\title{
BRIEF COMMUNICATION \\ Room-temperature production of silver-nanofiber film for large-area, transparent and flexible surface electromagnetic interference shielding
}

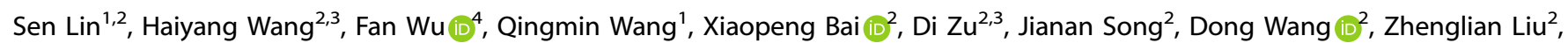
Ziwei $\mathrm{Li}^{2}$, Nian Tao ${ }^{1}$, Kai Huang $\mathbb{D}^{2}$, Ming $\mathrm{Lei}^{1}$, Bo $\mathrm{Li}^{3}$ and Hui $\mathrm{Wu}^{2}$

A kind of pollution known as electromagnetic interference (EMI), which results from ubiquitous usage of various electronic communication and military radar equipment, has been receiving increasing attention recently. However, large-area EMI shielding on transparent and/or curved surfaces, including building windows, curved glass wall, and special requirements spaces (SRSs), remains hard to achieve. In this paper, a silver nanofiber (AgNF) based flexible and transparent EMI shielding film was successfully assembled via a room-temperature roll-to-roll production method. For transparent application scenario, AgNF with $89 \%$ transmittance in visible range and $1 \mu \mathrm{m}$ thickness shows $\sim 20 \mathrm{~dB}$ shielding efficiency (EMI SE). On the other hand, total shielding $(>50 \mathrm{~dB})$ is obtained when the thickness of AgNF increases to $10 \mu \mathrm{m}$, while its transmittance in visible range remains higher than $75 \%$. Considering the facile and scale-free production technology, this material can be readily applied in large-scale, transparent, and/or SRSs EMI shielding.

npj Flexible Electronics (2019)3:6; https://doi.org/10.1038/s41528-019-0050-8

\section{INTRODUCTION}

While various modern electronic equipment in many fields are improving people's daily life, a kind of pollution known as electromagnetic radiation or electromagnetic interference (EMI) emerged. Among the concerned wave ranges, $\mathrm{X}$-band electromagnetic wave with the frequency from 8 to $12 \mathrm{GHz}$ plays an important role in military, weather radar, TV picture transmission, mobile device and telecommunication fields. ${ }^{1,2}$ This issue, however, if left unchecked can cause severe adverse effects to communication systems, sophisticated electronics and even human bodies. ${ }^{3-7}$ Foreseeably, EMI shielding will become more and more important for modern society due to the rapid growth of radio frequency radiation sources. ${ }^{8,9}$ The primary mechanism of EMI shielding is reflection. For reflecting the radiation by shielding materials, the shielding materials must have mobile charge carriers (electrons/holes) which interact with the electromagnetic fields in the radiation. ${ }^{10}$ The auxiliary mechanism of EMI shielding requires the absorption of electromagnetic radiation because the electrical and/or magnetic dipoles of the material interact with the radiation. High conductivity is the main factor that determines the reflectivity and absorption characteristics of shielding. ${ }^{11}$

The earliest method of preventing EMI was using a specific slurry, in which metal powders such as silver, copper and nickel are mixed into a diluent. The drawbacks of this method include poor binding force, low EMI shielding efficiency (EMI SE) and powder nonuniformity, seriously restricting its further development. Metal sheet made by electroless plating, vacuum deposition, and sputtering is another type of material used for EMI shielding. ${ }^{12-15}$ However, electromagnetism properties of these bulky materials are susceptible to cracks come from bending and stretching, which determine that metal sheets cannot be used in curve-surface and flexible situations. Recently, various nanomaterials have been developed to resist static hazard and electromagnetic wave pollution, including nano-metal, ${ }^{16-19}$ carbon, $^{20-22}$ and polymer-supported composites. ${ }^{23-30}$ Albeit these materials with favorable EMI SE can be successfully applied to curve-surface coating and equipment casing, there has been little progress with EMI shielding of windows, since in this case sufficient shielding, optical transparence and dimensions have to be achieved.

It is generally agreed that the effectiveness of a reflection-type shielding material requires a continuous conductive structure with an electrical conductivity of at least $1 \mathrm{~S} \mathrm{~m}^{-131}$. As a good conductor, silver-based materials can easily meet and even exceed this requirement. However, for large-scale and transparent surface applications, an ineluctable contradiction is that in-plane continuous silver layer shows highly visible transmittance only if its thickness is far below skin depth ( 650 nm for silver materials). Therefore, zero-dimensional silver nanoparticles coating material and two-dimensional silver film hardly to be considered as EMI shielding materials. On contrary, one-dimensional (1D) silver network is a promising structure to optimize the trade-off between transmittance and thickness due to its intrinsic low fillfactor and adjustable diameter. In recent years, more and more

\footnotetext{
${ }^{1}$ State Key Laboratory of Information Photonics and Optical Communications and School of Science, Beijing University of Posts and Telecommunications, 100876 Beijing, China;

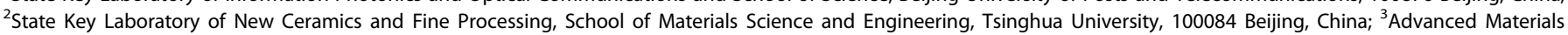

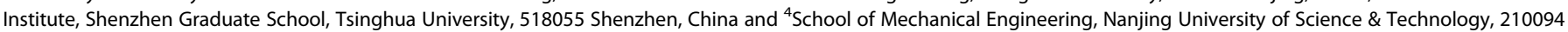
Nanjing, China

Correspondence: Ming Lei (mlei@bupt.edu.cn) or Bo Li (boli@mail.tsinghua.edu.cn) or Hui Wu (huiwu@tsinghua.edu.cn)
}

Received: 4 September 2018 Accepted: 10 January 2019

Published online: 12 March 2019 

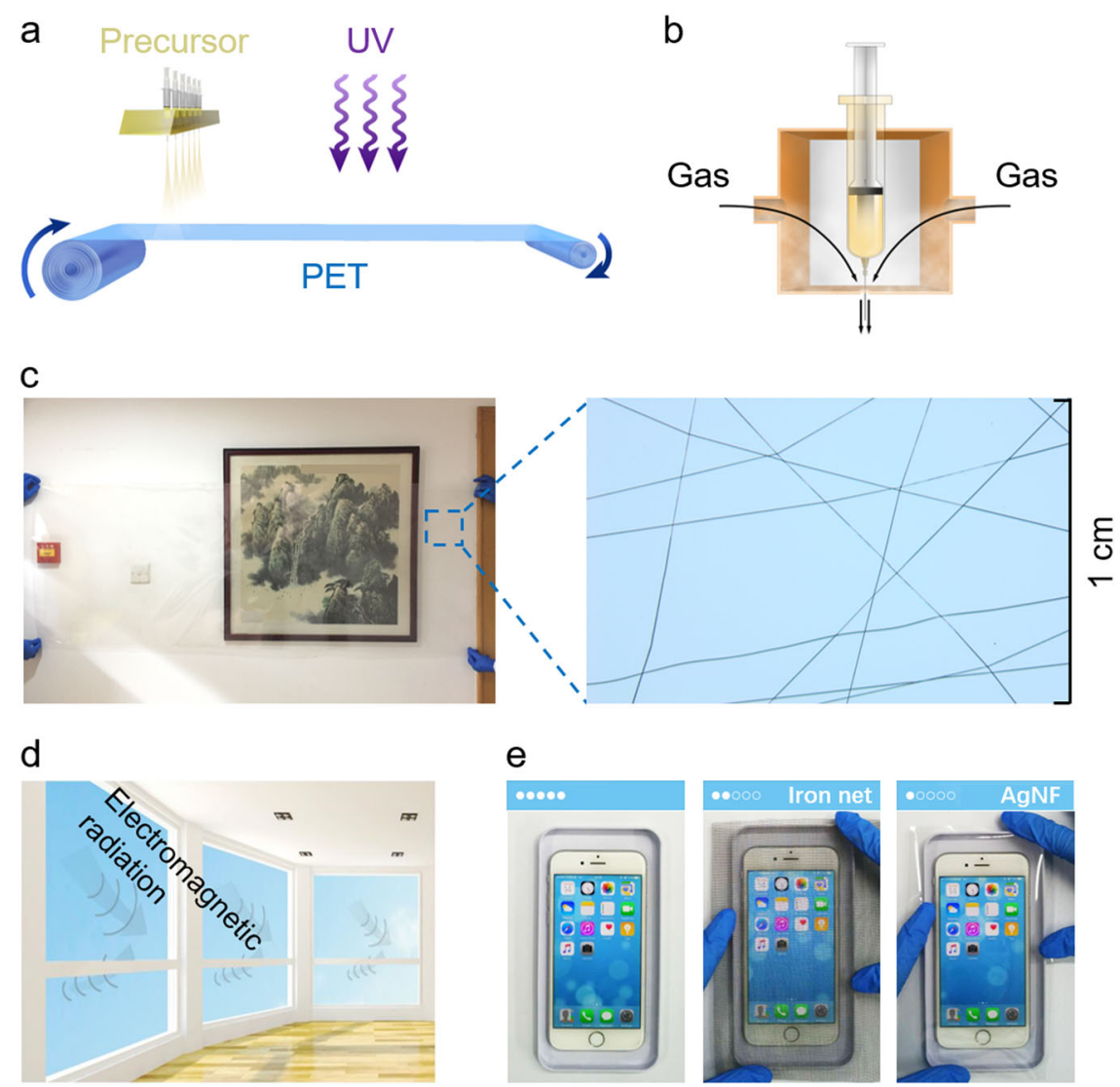

Fig. 1 Schematic of blow-spinning and material exhibition. a Fabrication of AgNF includes continuous blow-spinning and in-situ UV irradiation. b Partial enlarged detail of needle module shows the principle of blow-spinning. c A photograph of a window-scale ( $3 \mathrm{~m} \times 1 \mathrm{~m})$ AgNF EMI shielding film and its optical microscope image, $\mathbf{d}$ which could be considered as an EMl shielding functional material in modern architecture with large expanse of glass. e An EMI shielding demo consist of a grounded metal salver and different cover films include iron net and AgNF/PET

technologies have been proposed for continuous and large-scale fabrication of metal fibers and networks. ${ }^{32-37}$ Beyond solution reduction, ${ }^{38,39}$ successive multistep growth (SMG) method, ${ }^{40}$ and electrostatic spinning, ${ }^{41}$ 1D silver include silver nanowires (AgNWs), ${ }^{42-45}$ silver nanotroughs, ${ }^{46}$ and silver fiber $\left(\right.$ AgNF ${ }^{47}$ can be more cost-effectively fabricated in this day. Large-scale and room-temperature production of 1D silver is what people always pursuing. The challenge mainly consist in the annealing process for the reduction of silver ion, or the spattering technology for directly depositing of metallic silver, which are usually performed in a confined space such as a tube furnace or a sputtering apparatus. Such confined equipments will directly limit the scale of the final products. ${ }^{48}$ Anothoer key issues to be resolved is that room-temperature junction-fusing between crossing 1D silver network during fabrication process, which can greatly enhance material conductivity.

In this work, we fine investigate the technological parameter of blow-spinning, and propose a large-scale and transparent junction interconnected AgNF network for transparent surfaces SMI shielding. The AgNF network is fabricated via a roll-to-roll (R2R) production combining blow-spinning with in-situ UV irradiation. ${ }^{48}$ The forming of junction between crossing fibers is achieved by incorporation of lauryl sodium sulfate (SDS) to decrease precursor solution surface tension and adjusting of gas speed of blowspinning. High transmittance in visible range and diameter larger than silver's skin depth was obtained by finely adjusting other technological parameters such as spinning time, polymer concentration and needle type.

\section{RESULTS}

Figure 1a shows a simplified schematic of roll-to-roll blowspinning process and UV treatment. Using this air-driven method (Fig. 1b), a fiber network can be easily fabricated with arbitrary size to cater to different environments, from portable device to building-scale applications, while maintaining the continuous structure of each fiber (Fig. 1c, d and S1, Supplemental Material). For the protection of precision instruments and portable devices, we designed an EMI shielding demo consisting of a grounded metal salver and different cover films (iron net and AgNF/PET). As shown in Fig. 1e, the signal strength dropped to about $40 \%$ when the mobile phone was covered by an iron net. When the cover was changed to AgNF/PET film, there was only about $20 \%$ signal left.

In a typical blow-spinning production, solution viscosity and needle type codetermine the diameter of final fibers, injection speed and air velocity codetermine the productivity. The transmittance of fiber network is codetermined by sample thickness, fibers density (so called fill-factor) and fibers diameter. The thickness of fiber network is in linear relation to the spinning time, and there is a negative correlation between the thickness of fiber samples and its optical transmittance in the visible region. Therefore, by controlling the spinning time and fibers diameter, the optical transmittance of AgNF can be varied (Fig. 2a, b). As an $X$-band EMI shielding material, AgNF with the diameter larger than $650 \mathrm{~nm}$ is demanded as mentioned before. By co-adjusting needle type and concentration of PVP, AgNF with the diameter from $<500$ to $>1000 \mathrm{~nm}$ can be obtained. Wherein, G30 needle with an inner diameter of $0.16 \mathrm{~mm}$ and a PVP concentration of $5 \%$ are chosen to 
a

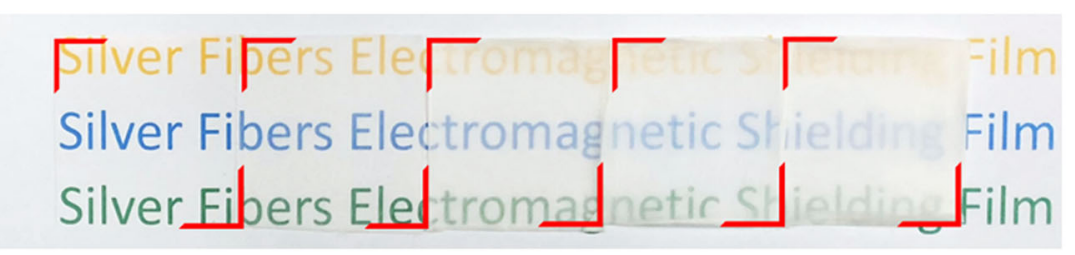

b

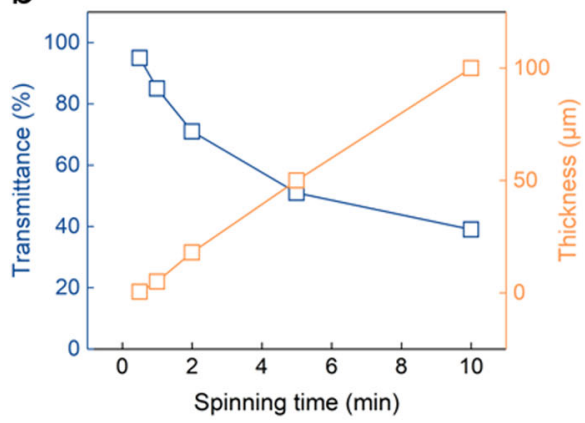

C

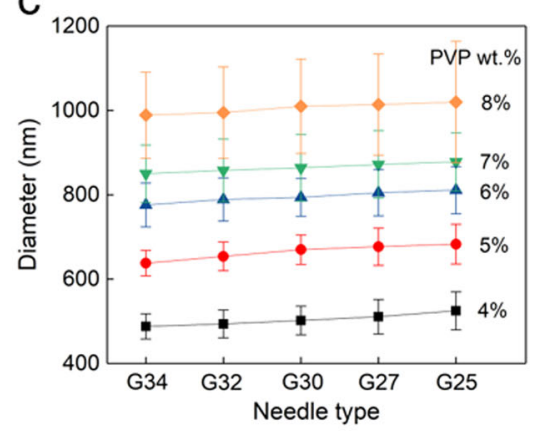

d
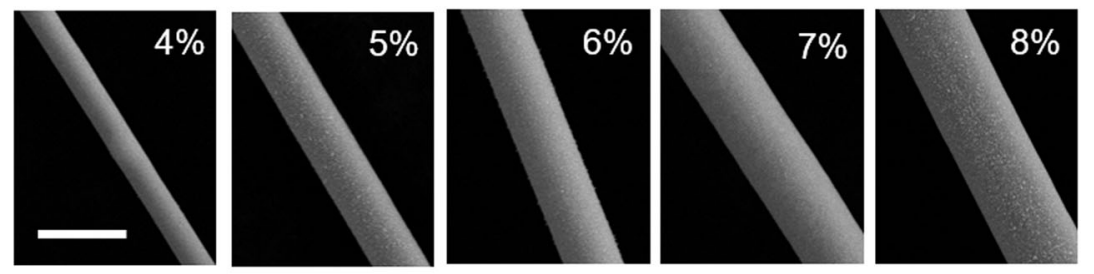

e

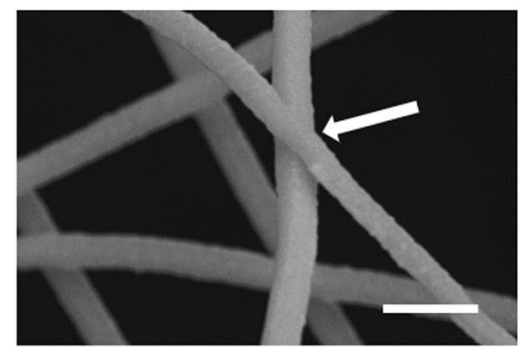

f

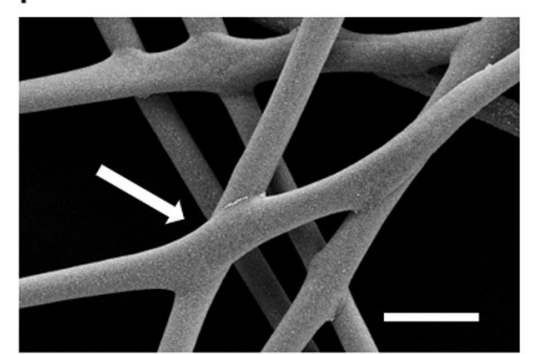

Fig. 2 Optical, crystallographic, mechanical and micromorphological characteristic of AgNF. a A photograph of as-prepared fiber film after different spinning times (from left: 1, 2, 5, 10, and $20 \mathrm{~min}$ ). b Correlation of technological parameter include transmittance and fiber thickness versus spinning time of blow-spinning. c Diameter of AgNF versus needle type. Corresponding inner diameter: $0.06 \mathrm{~mm}$ (G34), $0.11 \mathrm{~mm}$ (G32), $0.16 \mathrm{~mm}$ (G30), $0.21 \mathrm{~mm}$ (G27), and $0.26 \mathrm{~mm}$ (G25). d SEM of AgNF with different PVP concentration spun with G30 needle. Scale bar: $1 \mu \mathrm{m}$. e, $\mathbf{f}$ SEM images of AgNF and junction interconnected AgNF. Scale bar: $1 \mu \mathrm{m}$

spin $\sim 650 \mathrm{~nm}$ AgNF (Fig. 2d). Moreover, the combination of each single AgNF in network structure will severely affect its conductivity, $^{49}$ and then further affect its electromagnetism property. SEM images of AgNF without and with the adding of sodium dodecyl sulfate (SDS) are shown in Fig. 2e, $\mathrm{f}$, respectively. The junctions of AgNF with SDS naturally interconnected after $3 \mathrm{~h}$ UV irradiation. This junction interconnected structure mainly comes from the coefficient of surface tension reducing after SDS is added into the precursor solution, and the squeezing action of high-speed airflow on deposited precursor fibers, with is ductile because of the residual organic solvent.

Research has shown that EMI shielding materials usually need high conductivity of at least $1 \mathrm{~S} \mathrm{~m}^{-1} .31$ The fiber conductivity increases continuously with time under UV irradiation. Three hours of irradiation UV led to an equivalent result of annealing at $240^{\circ} \mathrm{C}$, after that precursor fibers completely reduce to silver, which then becomes an excellent conductor (Fig. 3a, b). Raman spectrum demonstrates the reaction of $\mathrm{Ag}^{+}$ion (Fig. 3c, d). At the Raman shift about $1039 \mathrm{~cm}^{-1}$ was decreased after $3 \mathrm{~h}$ of UV irradiation, which attributing to $\mathrm{NO}^{3-}$ ion. $\mathrm{C}-\mathrm{C}$ bond at $1428 \mathrm{~cm}^{-1}$ from PVP was also decreased after $3 \mathrm{~h}$ of UV irradiation. Moreover, two characteristic peaks around 1350 and $1553 \mathrm{~cm}^{-1}$ were found, suggesting the existent of amorphous carbon coating (Fig. 3e). Under such a thin amorphous carbon layer, silver particles on fiber are uniform and consecutive (Fig. 3f, $\mathrm{g}$ and S2, Supplemental Material). XPS results of AgNF in Ag3d region showed well separated spin-orbit components with a gap of $\sim 6.0 \mathrm{eV}$, corresponding to silver metal. Peak differentiation imitating results of $\mathrm{C} 1 \mathrm{~s}$ and $\mathrm{O} 1 \mathrm{~s}$ also indicate the same reaction process during UV irradiation (Figure S3, Supplemental Material). ${ }^{4,50}$ Figure $3 \mathrm{~h}-\mathrm{j}$ show the results of flexibility and durability test of AgNF and Ag films. As a metal-based fiber material, AgNF shows both high flexibility and bending stability (Fig. 3h). Compared with the Ag film obtained via magnetron sputtering, AgNF shows superior 


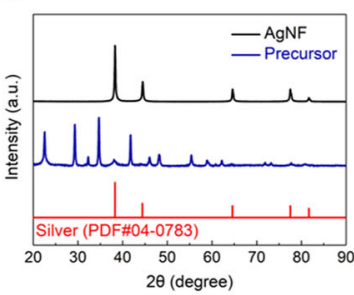

C

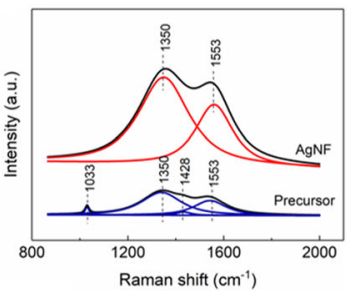

e

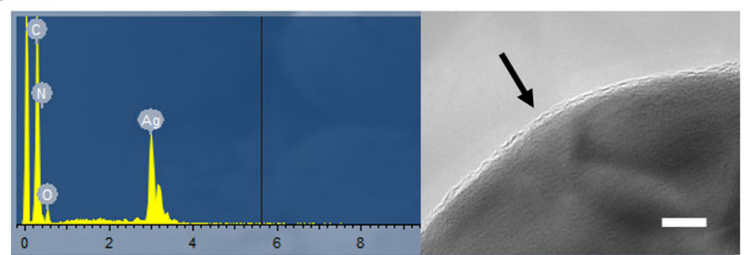

b

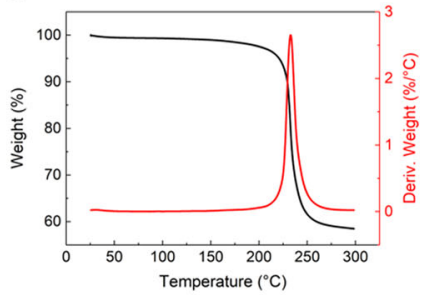

d

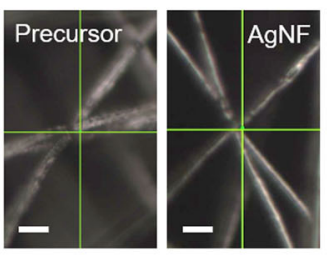

f

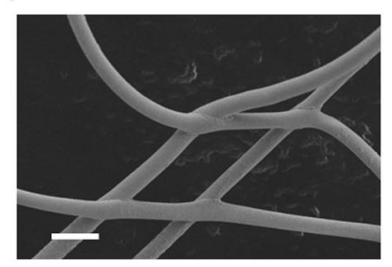

g

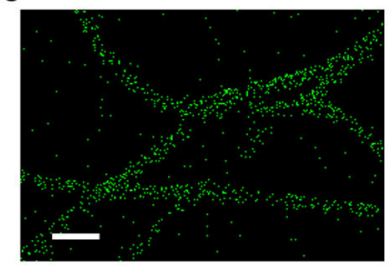

h
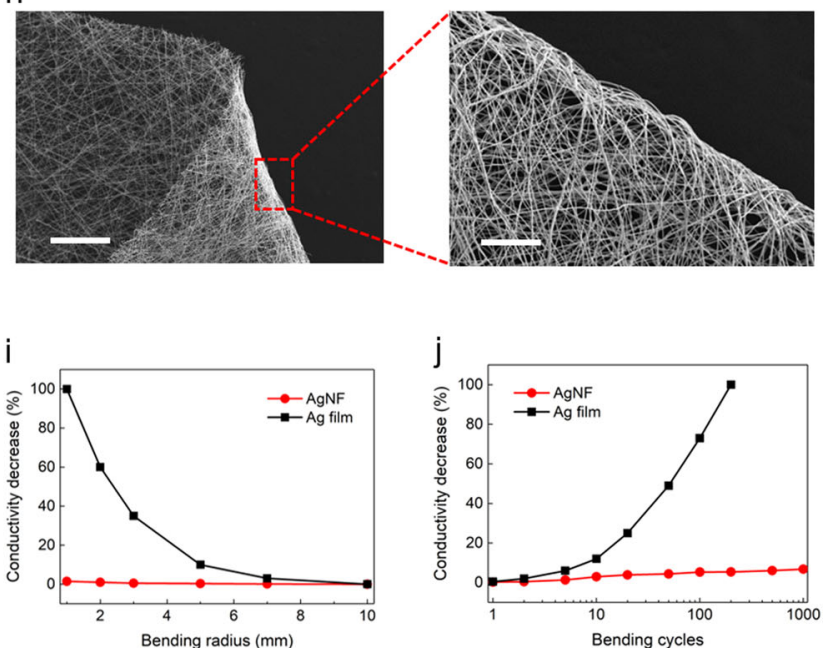

Fig. 3 Chemical, crystallographic, and mechanical properties of AgNF. a XRD spectra of as-prepared AgNF and precursor fibers, red line shows the peaks position of silver JCPDS card No. 04-0783. b TGA and DSC results of precursor fibers. c Raman spectra of precursor fiber and AgNF from 850 to $2000 \mathrm{~cm}^{-1}$. d Selected area SEM image of precursor fiber, and AgNF. Scale bar: $1 \mu \mathrm{m}$. e-g Element energy spectrum of AgNF. Inset of e shows the TEM image of graphite carbon layer. Scale bar: $5 \mathrm{~nm}$. $\mathbf{f}, \mathbf{g}$ SEM and element mapping of silver of AgNF. Scale bar $1 \mu \mathrm{m}$. $\mathbf{h}$ SEM image of a bended AgNF film and its partial enlargement. Scale bar: 300 and $50 \mu \mathrm{m}$. i Durability test showing conductivity decrease versus bending radius, and $\mathbf{j}$ bending cycles of AgNF on PET and sputtered silver film on Pl, which were both bent to a radius of $4 \mathrm{~mm}$ for a thousand times

mechanical properties include the ultralow bending radius (Fig. 3i) and 1000 times bending durability (Fig. 3j), which as the result of two characteristics: (i) the specific structural advantage of onedimensional materials (Figure S4, Supplemental Material); (ii) the inherent flexibility of metallic silver. The bendability of AgNF enables applying this material on curved surfaces, such as certain windows.

In addition to its remarkable transmittance and flexibility, AgNF also shows good microwave shielding performance. The EMI SE is a key index in EMI shielding related fields, and it can be expressed based on the $S$-parameters according to following equations: ${ }^{51}$

$\mathrm{SE}_{\mathrm{T}}=\mathrm{SE}_{\mathrm{R}}+\mathrm{SE}_{\mathrm{A}}=10 \log \left(\frac{1}{\left|\mathrm{~S}_{21}\right|^{2}}\right)$

$S E_{R}=10 \log \left(\frac{1}{1-\left|S_{11}\right|^{2}}\right)$

$\mathrm{SE}_{\mathrm{A}}=10 \log \left(\frac{1-\left|S_{11}\right|^{2}}{\left|S_{21}\right|^{2}}\right)$

As shown in Fig. 4a, a comparison was made between AgNF with a density of $0.2 \mathrm{~g} \mathrm{~cm}^{-3}$ and other EMI shielding materials, including CNT, ${ }^{19,52,53} \mathrm{rGO}^{20,54}$ graphene, $^{21,55-58}$ stainless steel fiber, ${ }^{7}$ silver NW, ${ }^{16}$ copper NW, ${ }^{17}$ nickel hybrid fiber, ${ }^{7,59}$ carbon fiber, ${ }^{7}$ and some ceramic materials. ${ }^{59-61}$ Moreover, the specific EMI SE of AgNF is orders of magnitude higher than that of graphene, ${ }^{22,62}$ copper, ${ }^{63}$ stainless steel, ${ }^{63}$ nickel fiber, ${ }^{63}$ carbon fiber, ${ }^{63}$ and CNT. ${ }^{20}$ Owing to the low density of fiber structure, ${ }^{37}$ ultralight AgNF film the specific EMI SE of AgNF reach $\sim 5000 \mathrm{~dB} \mathrm{~cm}^{3} \mathrm{~g}^{-1}$, which is higher than most EMI shielding material have been used (Fig. 4b). Figure 4c shows the spectra between 8 and $12 \mathrm{GHz}$ of AgNF for different thickness from 100 to $1 \mu \mathrm{m}$. It can be seen that the thickness of AgNF is a critical parameter for EMI shielding, with $100 \mu \mathrm{m}$ AgNF film resulting in an EMI SE about $76 \mathrm{~dB}$. Moreover, this value decreases with decreasing thickness. Results from the $S$ parameter, including $\operatorname{EMI~SE}_{\mathrm{R}}\left(\left|S_{21}\right|\right)$ and $\operatorname{EMI~SE}_{\mathrm{A}}\left(\left|S_{11}\right|\right)$ (Fig. 4d, e) further confirmed the reflectivity of the AgNF film. The theoretical EMI SE values can be calculated using plan wave theory based on the measured electrical conductivity of AgNF (Figure S5, Supplemental Material). ${ }^{34}$ While $\sigma / \omega \varepsilon_{0} \gg 0$, AgNF can be identified as a good-conductor and its EMI SE calculated according to following equations: ${ }^{64,65}$

$$
\mathrm{SE}=10 \log \left[\frac{1}{4}\left\{\begin{array}{c}
\frac{\sigma}{2 \omega \varepsilon_{0}}\left[\cosh \left(\frac{2 d}{\delta}\right)-\cos \left(\frac{2 d}{\delta}\right)\right]+2 \sqrt{\frac{\sigma}{2 \omega \varepsilon_{0}}}\left[\sinh \left(\frac{2 d}{\delta}\right)+\sin \left(\frac{2 d}{\delta}\right)\right] \\
+2\left[\cosh \left(\frac{2 d}{\delta}\right)+\cos \left(\frac{2 d}{\delta}\right)\right]
\end{array}\right\}\right],
$$

$\delta=\sqrt{\frac{2}{\mu_{0} \omega \sigma}}$

where $\sigma$ is the conductivity of material, $\omega$ is the angular frequency of electromagnetic wave, $\varepsilon_{0}$ is the permittivity of vacuum, $\mu_{0}$ is the permeability of vacuum, $d$ is the sample thickness, and $\delta$ is the skin depth of materials. As shown in Figure S6, Supplemental Material, the theoretical EMI SE values are slightly higher than the measured ones, owing to the structure differences between inplane network and layered composites. The flexibility is an 
a

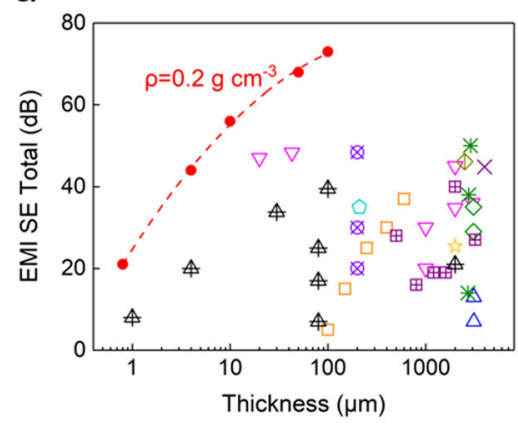

- $\mathrm{AgNF}$

田 CNT

4 rGO

$\nabla$ Graphene

$\diamond$ Stainless steel fibe

$\square$ AgNW

- CuNW

$\triangle \mathrm{Ni}$ fiber

* Carbon wires

๑) $\mathrm{PdTiO}_{3}$

$\times$ YIG

$\mathrm{Si}_{3} \mathrm{~N}_{4}$ b

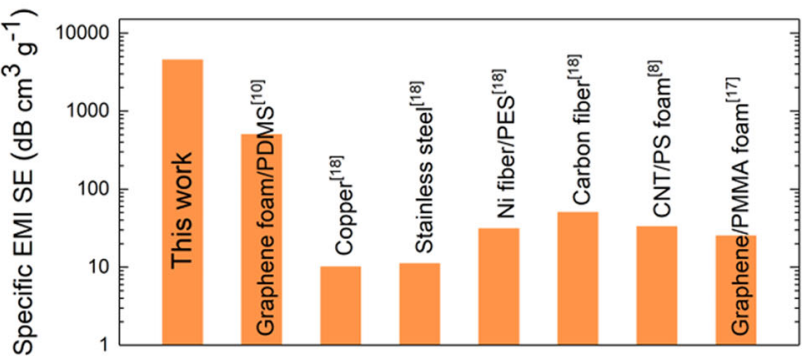

C

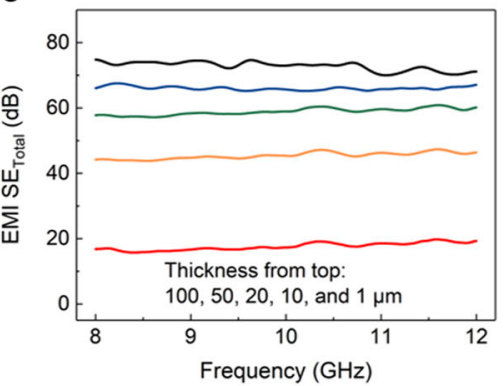

f

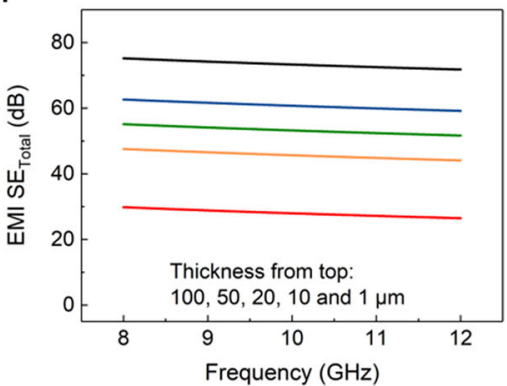

g

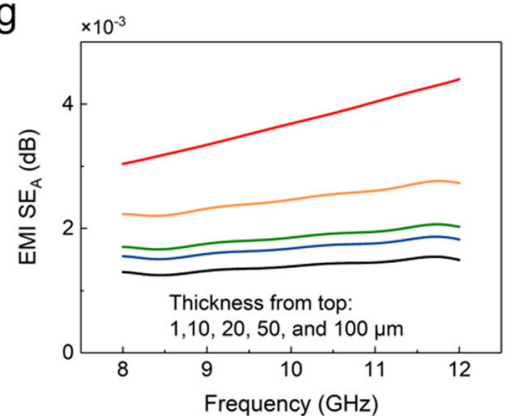

d

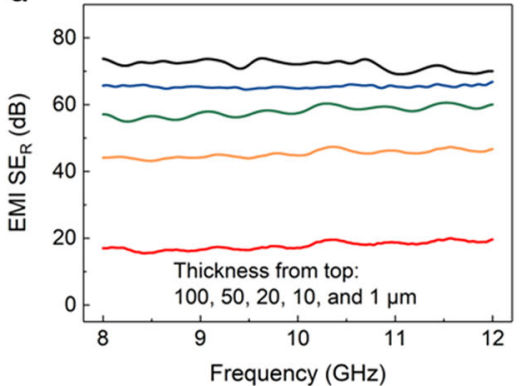

h

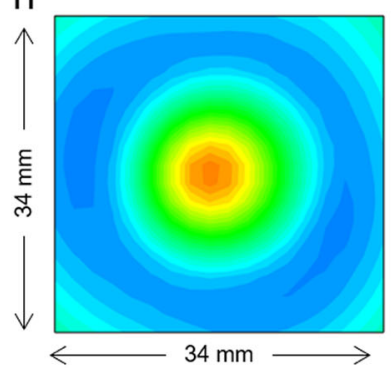

j

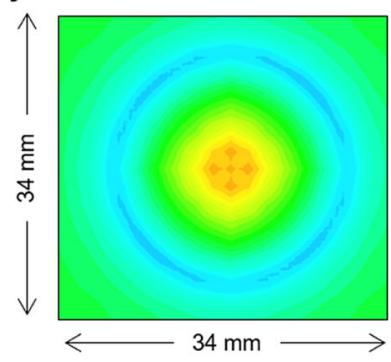

e
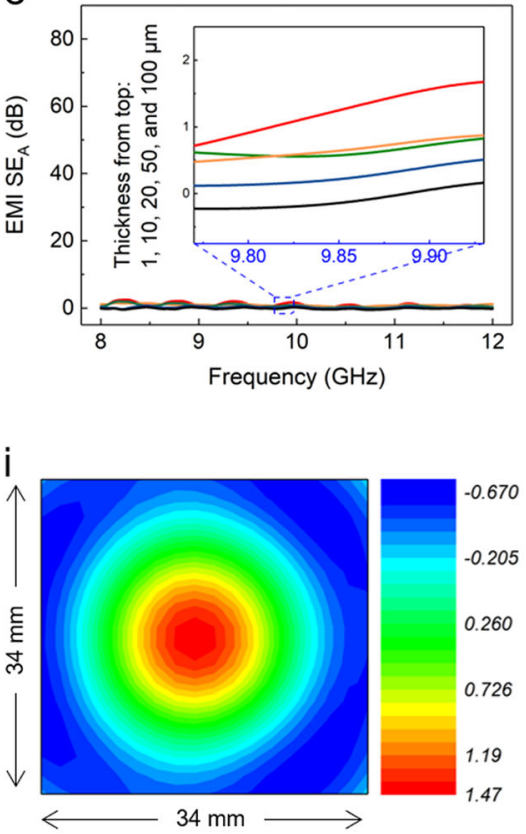

k

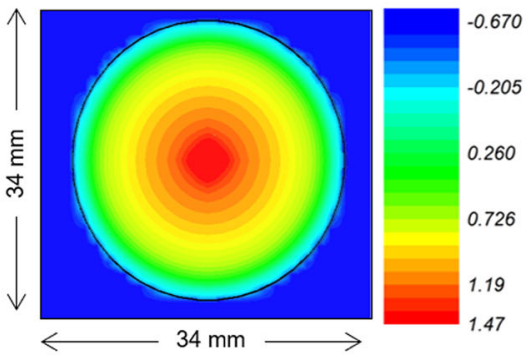

Fig. 4 EMI shielding performance: VNA experimental results, simulation and field scanning. a SMI SE density of $0.2 \mathrm{~g} \mathrm{~cm}^{-3}$ and other EMI shielding films from references. b Specific EMI SE of AgNF and other EMI shielding materials from references. c EMI SE $E_{\text {Total }}$ of AgNF and other common materials from 8 to $12 \mathrm{GHz}$. d, e EMI SE $\mathrm{R}_{\mathrm{R}}$ and EMI SE $\mathrm{S}_{\mathrm{A}}\left(\left|\mathrm{S}_{21}\right|\right.$ and $\left.\left|S_{11}\right|\right)$ versus frequency (from 8 to $12 \mathrm{GHz}$ ) of AgNF with different thicknesses. Time domain simulation based on a metallic-silver-network approximate model include f, g EMI SE $E_{\text {Total }}$ and EMI SE $\left(\left|\mathrm{S}_{21}\right|\right.$ and $\left.\left|S_{11}\right|\right)$ versus frequency (from 8 to $12 \mathrm{GHz}$ ) of AgNF with different thicknesses. Field scanning of a point source $\mathbf{h}$ without and $\mathbf{i}$ with an annular AgNF sheet at the emission frequency of $10 \mathrm{GHz}$. Field simulation of a point source $\mathbf{j}$ without and $\mathbf{k}$ with a copper sheet at the emission frequency of $10 \mathrm{GHz}$

important character of EMI shielding materials. Highly flexible is one of the most important premise for mass production and transport, which also show significance in many fields such as curve-surface shielding. Figure S7, Supplemental Material, shows the EMI shielding performance of $100-\mu \mathrm{m}$-thick AgNF network on different bending stages from its plane state to the bending radius of $3.0 \mathrm{~cm}$. The EMI SE of AgNF network shows slight increase with the reduction of bending radius, owing to more material was filled in to cover the cross section of waveguide when bending radius decrease.

In order to describe the working principle of the AgNF network structure and the behavior of plane electromagnetic wave during 
EMI shielding, time domain simulation was performed in computer simulation technology microwave studio (CST MWS) based on a metallic-silver-network approximate model. The EMI $\mathrm{SE}_{\mathrm{R}}\left(\left|S_{21}\right|\right)$ and $\mathrm{EMI} \mathrm{SE}_{\mathrm{A}}\left(\left|S_{11}\right|\right)$ results are shown in Fig. 4f, $g$, respectively, and show agreement with the VNA experimental results. Noted that the simulated result of $S_{|21|}$ is higher than that of test result, which mainly due to the uneven thickness of singlelayer AgNF. As AgNF thickness decreases nearby skin depth, the diameter of singe fiber directly determined the local thickness. As shown in Fig. 2c, red line and error bar, some fibers diameter may drop below $650 \mathrm{~nm}$, which will led to a local electromagnetic transmission. Therefore, it can be concluded that the AgNF network, when its thickness exceeds skin depth, behaves as a simple metallic-silver-network structure in $8-12 \mathrm{GHz}$ microwave frequency band. The field distribution through structural unit is shown in Figure S8, Supplemental Material, with the incident wave propagating onto the structure unit from the right. Results show that total shielding for fill-factor of metallic-silver-network exceeds $20 \%$, which is according well with the experimental results of AgNF. Field distribution of plane incident wave through different fill-factor AgNF of 20,44, 55, and $80 \%$ (corresponding to the fiber thicknesses of $10,20,50$, and $100 \mu \mathrm{m}$ ) are also simulated via CST MWS (Figure S9, Supplemental Material). A typical surface reflection can be found since AgNF with a fill-factor of $20 \%$. To investigate its microwave blocking effect visually, point source field scanning was performed on an annular AgNF sheet (Fig. 4h, i), and subsequently performed a simulation with a $1 \mathrm{~mm}$ copper sheet under the same conditions (Fig. 4 j, k). Results show a similar field distribution (total shielding), indicating an equivalent microwave shielding effect of the low fill-factor AgNF network and copper.

\section{DISCUSSION}

In conclusion, a flexible and transparent AgNF network structure was continuous fabricated via a R2R production combining blowspinning with in situ UV irradiation. By controlling the spinning time, AgNF networks with different thicknesses and fill-factors were obtained. EMI SE was discussed in the range of $8-12 \mathrm{GHz}$. VNA experimental results demonstrate a remarkable EMI SE of AgNF, up to $\sim 76 \mathrm{~dB}$ of $\mathrm{EMI} \mathrm{SE}_{\mathrm{R}}\left(\left|S_{21}\right|\right)$ with a thickness of $100 \mu \mathrm{m}$. By comparison with the CST simulation results, we found that the shielding behavior of AgNF (when $d \gg \delta$ ) is very similar to that of simple metallic-silver-network and both of them exhibited total shielding for fill-factors higher than $20 \%$. Moreover, it was found that the shielding effect of $100 \mu \mathrm{m}$ AgNF for microwaves is equivalent to a $1 \mathrm{~mm}$ copper sheet. Therefore, we can conclude that remarkable EMI SE, unique flexibility and adjustable transparence at visible light of AgNF enable it to be applied for large-scale or complex transparent surface EMI shielding.

\section{METHODS}

\section{Materials}

$\mathrm{AgNO}_{3}$ (99.8\%) was purchased from Beijing Chemical Works. PVP (Mw $=$ $1,300,000)$ and Sodium dodecyl sulfate (SDS, 99.0\%) were purchased from Alfa Aesar. Acetonitrile (99.0\%) was purchased from Aladdin. Fluorocarbon surfactant (Capstone FS-3100) was purchased from Shanghai Jianbang Industry Co., Ltd. PET with a thickness of $0.1 \mathrm{~mm}$ is commercial available. All reagents were used without further purification.

\section{Preparation of AgNF network}

In this work, we used a R2R production to prepare PET-based AgNF network. Typically, $1.975 \mathrm{~g}$ of PVP and $16.120 \mathrm{~g}$ of $\mathrm{AgNO}_{3}$ were dissolved in $21.400 \mathrm{~g}$ of acetonitrile. Then, $1 \mathrm{wt} \%$ of SDS was added into the solution to decrease surface tension to facilitate forming junctions between crossing fibers. A pale-yellow precursor solution was obtained after $30 \mathrm{~min}$ of stirring. For blow spinning, the precursor solution was jetted out from four air-propelled injectors to a rolling continuous track covered with PET. The speed of the continuous track was $0.5 \mathrm{~m} \mathrm{~h}^{-1}$ and the speed of injection was $1.0 \mathrm{~mL} \mathrm{~h}^{-1}$. Four UV lamps ( $250 \mathrm{~W}$, Philips Lighting) located side by side were used to reduce $\mathrm{Ag}^{+}$. The distance between the UV light source and substrate was set to $10 \mathrm{~cm}$. The AgNF based EMI shielding film was obtained after $3 \mathrm{~h}$ of UV irradiation.

\section{Material microscopic characterization}

The micro-morphological characterization of AgNF were measured via a field emission scanning electron microscope (FE-SEM, LEO-1530, Zeiss, Germany) and a transmission electron microscope (TEM, JEOL-2100, JEOL Ltd. Japan). The crystal lattice information of samples were obtained via an X-ray diffractometer (XRD, D/max 2500, Rigaku, Japan) with Cu Ka radiation $\left(\lambda=1.54178 \AA\right.$ ) from $2 \theta=10^{\circ}$ to $90^{\circ}$. Raman spectra were obtained via a Raman spectrometer (LabRAM HR Evolution, HORIBA Jobin Yvon, French) with $325 \mathrm{~nm}$ excitation wavelength. An X-ray photoelectron spectrometer (Escalab 250Xi, Thermo Fisher, America) equipped with an Al Ka radiation source $(1487.6 \mathrm{eV})$ and hemispherical analyzer with a pass energy of $30.00 \mathrm{eV}$ and an energy step size of $0.05 \mathrm{eV}$ was used. Thermogravimetric analysis and differential thermal gravity data for the measurement of PVP were obtained via a thermogravimetric analyzer (STA 449 F3, Jupiter, Germany). The transmittance of AgNF in visible range was collected via an UV-Vis-NIR spectroscope (Lambda 950, Perkin-Elmer, America) with quartz glass as reference. The sheet resistance of as-prepared AgNF was measured via a multifunctional four-point probe tester (ST-2258C, Suzhou Jingge Electronic Co., LTD, China).

\section{EMI shielding characterization}

EMI SE of as-prepared materials were measured via a vector network analyzer (PNA-L N5230C, Agilent Technologie, America) with $8-12 \mathrm{GHz}$ calibration kit. Time domain simulation was performed in computer simulation technology microwave studio (CST MWS) based on a metallicsilver-network approximate model.

\section{ACKNOWLEDGEMENTS}

This study was supported by the National Basic Research of China (Grants 2015CB932500), National Natural Science Foundations of China (Grant 51661135025 and 51522207), BUPT Excellent Ph.D. Students Foundation (Grant GX2017205), and State Key Laboratory of Information Photonics and Optical Communications (Beijing University of Posts and Telecommunications).

\section{AUTHER CONTRIBUTIONS}

H.W. and S.L. conceived the idea and designed the research. S.L., H.Y.W., D.Z. prepared the materials. S.L., H.Y.W., X.P.B., D.W., J.A.S., Z.L.L., and Z.W.L. design the production technology. S.L., H.Y.W., X.P.B., D.Z., K.H., M.L., and H.W. conducted the micromorphological characterization of material. S.L., H.Y.W, F.W., and B.L. conducted the optical property measurement. S.L., F.W., Q.M.W., B.L., and H.W. conducted the EMI SE measurement and analyzed the data. S.L., and Q.M.W. conducted the plane electromagnetic wave calculation and simulation. S.L., H.Y.W., K.H., N.T., M.L., and H.W. conducted the mechanical tests. All authors contributed to the paper writing.

\section{ADDITIONAL INFORMATION}

Supplementary information accompanies the paper on the npj Flexible Electronics website (https://doi.org/10.1038/s41528-019-0050-8).

Competing interests: The authors declare no competing interests.

Publisher's note: Springer Nature remains neutral with regard to jurisdictional claims in published maps and institutional affiliations.

\section{REFERENCES}

1. Huang, Y. et al. The influence of single-walled carbon nanotube structure on the electromagnetic interference shielding efficiency of its epoxy composites. Carbon N. Y. 45, 1614-1621 (2007).

2. Pande, S. et al. Improved electromagnetic interference shielding properties of MWCNT-PMMA composites using layered structures. Nanoscale Res. Lett. 4, 327-334 (2009). 
3. Geetha, S., Kumar, K. K. S., Rao, C. R. K., Vijayan, M. \& Trivedi, D. C. EMI shielding: methods and materials-a review. J. Appl. Polym. Sci. 112, 2073-2086 (2009).

4. Zhang, Y. et al. Broadband and tunable high-performance microwave absorption of an ultralight and highly compressible graphene foam. Adv. Mater. 27, 2049-2053 (2015).

5. Paligova, M. et al. Electromagnetic shielding of epoxy resin composites containing carbon fibers coated with polyaniline base. Phys. A 335, 421-429 (2004).

6. Hakansson, E., Amiet, A. \& Kaynak, A. Electromagnetic shielding properties of polypyrrole/polyester composites in the $1-18 \mathrm{GHz}$ frequency range. Synth. Met. 156, 917-925 (2006).

7. Lee, S. et al. Smart contact lenses with graphene coating for electromagnetic interference shielding and dehydration protection. Acs Nano 11, 5318-5324 (2017).

8. Fernandez-Garcia, R. \& Gil, I. Measurement of the environmental broadband electromagnetic waves in a mid-size European city. Environ. Res. 158, 768-772 (2017).

9. Singh, A. P. et al. Encapsulation of gamma- $\mathrm{Fe}_{2} \mathrm{O}_{3}$ decorated reduced graphene oxide in polyaniline core-shell tubes as an exceptional tracker for electromagnetic environmental pollution. J. Mater. Chem. A 2, 3581-3593 (2014).

10. Chung, D. D. L. Electromagnetic interference shielding effectiveness of carbon materials. Carbon N. Y. 39, 279-285 (2001).

11. Shahzad, F. et al. Electromagnetic interference shielding with $2 D$ transition metal carbides (MXenes). Science 353, 1137-1140 (2016).

12. Jackson, B. C. \& Shawhan, G. Current review of the performance characteristics of conductive coatings for EMI control. 1998 IEEE Int. Symp. 1, 567-572 (1998).

13. Li, D. P., Goodwin, K. \& Yang, C. L. Electroless copper deposition on aluminumseeded ABS plastics. J. Mater. Sci. 43, 7121-7131 (2008).

14. Nagasawa, C., Kumagai, Y., Urabe, K. \& Shinagawa, S. Electromagnetic shielding particleboard with nickel-plated wood particles. J. Porous Mat. 6, 247-254 (1999).

15. Dixon, D. S. \& Masi, J. Thin coatings can provide significant shielding against low frequency EMF magnetic fields. 1998 IEEE Int. Symp. 1, 1035-1040 (1998).

16. Li, L. \& Chung, D. D. L. Electrical and mechanical-properties of electrically conductive polyethersulfone composites. Composites 25, 215-224 (1994).

17. Hu, M. J. et al. Flexible transparent PES/Silver nanowires/PET sandwich-structured film for high-efficiency electromagnetic interference shielding. Langmuir 28, 7101-7106 (2012).

18. Al-Saleh, M. H., Gelves, G. A. \& Sundararaj, U. Copper nanowire/polystyrene nanocomposites: Lower percolation threshold and higher EMI shielding. Compos Part A 42, 92-97 (2011).

19. Hoang, A. S. Electrical conductivity and electromagnetic interference shielding characteristics of multiwalled carbon nanotube filled polyurethane composite films. Adv. Nat. Sci. Nanosci. Nanotechnol. 2, 5766-5771 (2011).

20. Yang, Y. L. \& Gupta, M. C. Novel carbon nanotube-polystyrene foam composites for electromagnetic interference shielding. Nano Lett. 5, 2131-2134 (2005).

21. Yan, D. X. et al. Structured reduced graphene oxide/polymer composites for ultraefficient electromagnetic interference shielding. Adv. Funct. Mater. 25, 559-566 (2015).

22. Chen, Z. P., Xu, C., Ma, C. Q., Ren, W. C. \& Cheng, H. M. Lightweight and flexible graphene foam composites for high-performance electromagnetic interference shielding. Adv. Mater. 25, 1296-1300 (2013).

23. Yang, Y., Gupta, M. C. \& Dudley, K. L. Studies on electromagnetic interference shielding characteristics of metal nanoparticle- and carbon nanostructure-filled polymer composites in the Ku-band frequency. Micro Nano Lett. 2, 85-89 (2007).

24. Li, B. W., Shen, Y., Yue, Z. X. \& Nan, C. W. Enhanced microwave absorption in nickel/hexagonal-ferrite/polymer composites. Appl. Phys. Lett. 89, 132504 (2006).

25. Bagwell, R. M., McManaman, J. M. \& Wetherhold, R. C. Short shaped copper fibers in an epoxy matrix: their role in a multifunctional composite. Compos Sci. Technol. 66, 522-530 (2006)

26. Dhawan, S. K., Singh, N. \& Venkatachalam, S. Shielding behaviour of conducting polymer-coated fabrics in X-band, W-band and radio frequency range. Synth. Met. 0, 261-267 (2002).

27. Zhang, Y., Qiu, M. A., Yu, Y., Wen, B. Y. \& Cheng, L. L. A novel polyaniline-coated bagasse fiber composite with core-shell heterostructure provides effective electromagnetic shielding performance. ACS Appl. Mater. Interfaces 9, 809-818 (2017).

28. Lee, S. H. et al. Highly anisotropic Cu oblate ellipsoids incorporated polymer composites with excellent performance for broadband electromagnetic interference shielding. Compos Sci. Technol. 144, 57-62 (2017).

29. Ling, J. Q. et al. Facile preparation of lightweight microcellular polyetherimide/ graphene composite foams for electromagnetic interference shielding. ACS Appl. Mater. Interfaces 5, 2677-2684 (2013).

30. Yan, D. X. et al. Efficient electromagnetic interference shielding of lightweight graphene/polystyrene composite. J. Mater. Chem. 22, 18772-18774 (2012).

31. Thomassin, J. M. et al. Polymer/carbon based composites as electromagnetic interference (EMI) shielding materials. Mat. Sci. Eng. R. 74, 211-232 (2013).
32. Yu, Y., Yan, C. \& Zheng, Z. J. Polymer-assisted metal deposition (PAMD): a fullsolution strategy for flexible, stretchable, compressible, and wearable metal conductors. Adv. Mater. 26, 5508-5516 (2014)

33. Zhao, Z. Z. et al. Machine-washable textile triboelectric nanogenerators for effective human respiratory monitoring through loom weaving of metallic yarns. Adv. Mater. 28, 10267-10274 (2016).

34. $\mathrm{Yu}, \mathrm{Y}$. et al. Photoreactive and metal-platable copolymer inks for high-throughput, room-temperature printing of flexible metal electrodes for thin-film electronics. Adv. Mater. 28, 4926-4934 (2016).

35. Guo, R. S. et al. Biomimicking topographic elastomeric petals (E-petals) for omnidirectional stretchable and printable electronics. Adv. Sci. 2, 1400021 (2015)

36. Yu, Y., Zhang, Y. K., Li, K., Yan, C. \& Zheng, Z. J. Bio-inspired chemical fabrication of stretchable transparent electrodes. Small 11, 3444-3449 (2015).

37. Wang, H. L. et al. Ultralight, scalable, and high-temperature-resilient ceramic nanofiber sponges. Sci. Adv. 3, e1603170 (2017).

38. Jung, J. et al. Highly stretchable and transparent electromagnetic interference shielding film based on silver nanowire percolation network for wearable electronics applications. ACS Appl. Mater. Interfaces 9, 44609-44616 (2017).

39. Huang, Y. et al. A transparent, conducting tape for flexible electronics. Nano Res. 9, 917-924 (2016).

40. Lee, J. et al. Very long Ag nanowire synthesis and its application in a highly transparent, conductive and flexible metal electrode touch panel. Nanoscale 4 6408-6414 (2012).

41. Zhang, C. L. et al. Macroscopic-scale alignment of ultralong Ag nanowires in polymer nanofiber mat and their hierarchical structures by magnetic-fieldassisted electrospinning. Small 8, 2936-2940 (2012).

42. Hong, B. H., Bae, S. C., Lee, C. W., Jeong, S. \& Kim, K. S. Ultrathin single-crystalline silver nanowire arrays formed in an ambient solution phase. Science 294 348-351 (2001).

43. Sun, Y. G., Gates, B., Mayers, B. \& Xia, Y. N. Crystalline silver nanowires by soft solution processing. Nano Lett. 2, 165-168 (2002).

44. Sun, Y. G., Yin, Y. D., Mayers, B. T., Herricks, T. \& Xia, Y. N. Uniform silver nanowires synthesis by reducing AgNO3 with ethylene glycol in the presence of seeds and poly(vinyl pyrrolidone). Chem. Mater. 14, 4736-4745 (2002).

45. Caswell, K. K., Bender, C. M. \& Murphy, C. J. Seedless, surfactantless wet chemical synthesis of silver nanowires. Nano Lett. 3, 667-669 (2003).

46. $\mathrm{Wu}, \mathrm{H}$. et al. A transparent electrode based on a metal nanotrough network. Nat. Nanotechnol. 8, 421-425 (2013).

47. Huang, Y. et al. Large-scale spinning of silver nanofibers as flexible and reliable conductors. Nano Lett. 16, 5846-5851 (2016).

48. Lin, S. et al. Roll-to-roll production of transparent silver-nanofiber-network electrodes for flexible electrochromic smart windows. Adv. Mater. 29, 1703238 (2017).

49. Bai, X. P. et al. Continuous draw spinning of extra-long silver submicron fibers with micrometer patterning capability. Nano. Lett. 17, 1883-1891 (2017).

50. Xiong, Y. J. et al. Poly(vinyl pyrrolidone): a dual functional reductant and stabilizer for the facile synthesis of noble metal nanoplates in aqueous solutions. Langmuir 22, 8563-8570 (2006).

51. Wen, B. et al. Temperature dependent microwave attenuation behavior for carbon-nanotube/silica composites. Carbon N. Y. 65, 124-139 (2013).

52. Ajitha, A. R. et al. An effective EMI shielding material based on poly(trimethylene terephthalate) blend nanocomposites with multiwalled carbon nanotubes. New $\mathrm{J}$. Chem. 42, 13915-13926 (2018).

53. Chizari, K., Arjmand, M., Liu, Z., Sundararaj, U. \& Therriault, D. Three-dimensional printing of highly conductive polymer nanocomposites for EMI shielding applications. Mater. Today Commun. 11, 112-118 (2017).

54. Sawai, P., Chattopadhaya, P. P. \& Banerjee, S. Synthesized reduce graphene oxide ( $r G O)$ filled polyetherimide based nanocomposites for EMI Shielding applications. Mater. Today 5, 9989-9999 (2018).

55. Wang, G. L., Zhao, G. Q., Wang, S., Zhang, L. \& Park, C. B. Injection-molded microcellular PLA/graphite nanocomposites with dramatically enhanced mechanical and electrical properties for ultra-efficient EMI shielding applications. J. Mater. Chem. C 6, 6847-6859 (2018).

56. Sabira, K., Jayakrishnan, M. P., Saheeda, P. \& Jayalekshmi, S. On the absorption dominated EMI shielding effects in free standing and flexible films of poly (vinylidene fluoride)/graphene nanocomposite. Eur. Polym. J. 99, 437-444 (2018).

57. Liu, Y. H. et al. Graphene enhanced flexible expanded graphite film with high electric, thermal conductivities and EMI shielding at low content. Carbon N. Y. 133, 435-445 (2018).

58. Alam, F. E. et al. Highly conductive 3D segregated graphene architecture in polypropylene composite with efficient EMI shielding. Polymers 9, 662 (2017).

59. Pan, H. X., Yin, X. W., Xue, J. M., Cheng, L. F. \& Zhang, L. T. Microstructures and EM shielding properties of composite ceramics reinforced with carbon nanowires and nanowires-nanotubes hybrid. Ceram. Int. 43, 12221-12231 (2017). 
60. Dalal, J. et al. EMI shielding properties of laminated graphene and $\mathrm{PbTiO} 3$ reinforced poly (3,4-ethylenedioxythiophene) nanocomposites. Compos. Sci. Technol. 165, 222-230 (2018).

61. Choudhary, H. K. et al. Effect of coral-shaped yttrium iron garnet particles on the EMI shielding behaviour of yttrium iron garnet-polyaniline-wax composites. Chem. Select 3, 2120-2130 (2018).

62. Zhang, H. B., Yan, Q., Zheng, W. G., He, Z. X. \& Yu, Z. Z. Tough graphene-polymer microcellular foams for electromagnetic interference shielding. Acs Appl. Mater. Interfaces 3, 918-924 (2011).

63. Shui, X. P. \& Chung, D. D. L. Nickel filament polymer-matrix composites with low surface impedance and high electromagnetic interference shielding effectiveness. J. Electron Mater. 26, 928-934 (1997).

64. Colaneri, N. F. \& Shacklette, L. W. Emi shielding measurements of conductive polymer blends. IEEE Trans. Instrum. Meas. 41, 291-297 (1992).

65. Lee, C. Y. et al. Electromagnetic interference shielding efficiency of polyaniline mixtures and multilayer films. Synth. Met 102, 1346-1349 (1999).
Open Access This article is licensed under a Creative Commons Attribution 4.0 International License, which permits use, sharing, adaptation, distribution and reproduction in any medium or format, as long as you give appropriate credit to the original author(s) and the source, provide a link to the Creative Commons license, and indicate if changes were made. The images or other third party material in this article are included in the article's Creative Commons license, unless indicated otherwise in a credit line to the material. If material is not included in the article's Creative Commons license and your intended use is not permitted by statutory regulation or exceeds the permitted use, you will need to obtain permission directly from the copyright holder. To view a copy of this license, visit http://creativecommons. org/licenses/by/4.0/.

(c) The Author(s) 2019 